\title{
Consideraciones sobre el diagnóstico de COVID-19 y el papel del diagnóstico salival.
}

\author{
Considerations on the diagnosis of COVID-19 \\ and the role of the sallival diaginosis. \\ Perla Margarita Pacheco Morffi,* José Danilo Pacheco González, \\ Ana Belkys Hernández Millán, ${ }^{\S}$ Francisco Cázares de León"
}

\section{RESUMEN}

El nuevo coronavirus SARS-CoV-2 es el agente etiológico de la enfermedad por coronavirus de 2019 (COVID-19), convertida rápidamente en una pandemia, emergencia sanitaria y una crisis de salud pública en los países afectados a lo largo de los cinco continentes. El objetivo de la presente revisión bibliográfica fue describir algunas consideraciones sobre el diagnóstico del COVID-19 y el papel de los diagnósticos salivales. El diagnóstico molecular, la historia clínica, las manifestaciones clínicas, los hallazgos de laboratorio y los imagenológicos, y la prueba viral del ácido nucleico, el diagnóstico serológico, el sistema CRISPR/ Cas13 y la técnica SHERLOCK son elementos del arsenal diagnóstico de esta infección. Éstos son imprescindibles para el trabajo de los profesionales en salud, ya que generar y conocer medidas de identificación diagnóstica, son pilares esenciales, en el intento de mitigar una mayor propagación de esta infección.

Palabras clave: COVID-19, diagnóstico, diagnósticos salivales.

\section{ABSTRACT}

The novel coronavirus SARS-CoV-2 is the etiologic agent of the 2019 coronavirus disease (COVID-19), rapidly converted in a pandemic, sanitary emergency and Public Health crisis in the affected countries over the world. The objective of the present bibliographical review was to describe some considerations about the diagnosis of COVID-19 and the role of salivary diagnostics. The molecular diagnosis, the clinical history, the laboratory and imagenologic findings, the nucleic acid viral test, the serologic diagnosis, the CRISPR/Cas13 system, and the SHERLOCK technique, are all elements of the diagnostic arsenal to identify this infection. These elements are indispensables for the health professionals. Generating and knowing the diagnostic identification measures are essential pillars in the attempt of mitigating a greater propagation of this disease.

Keywords: COVID-19, diagnosis, salivary diagnostics.

\section{INTRODUCCIÓN}

L os coronavirus son virus ARN monocatenarios positivos pertenecientes a la familia Coronaviridae. Las infecciones humanas CoV son causadas por $\alpha-y$ $\beta$-CoVs. Se han descrito siete coronavirus con potencial de infección del ser humano, dos de ellos son graves:
Ios SARS coronavirus (SARS-CoV y SARS-CoV-2) y MERS coronavirus (MERS-CoV). El MERS-CoV (coronavirus del Síndrome Respiratorio del Medio Oriente), el SARS-CoV (coronavirus del síndrome respiratorio agudo severo), y el SARS-CoV-2, (coronavirus que causa la enfermedad por coronavirus del 2019, o COVID-19) son miembros de la familia de los $\beta$-CoVs. ${ }^{1-8}$

\footnotetext{
* Estudiante de cuarto año de la Carrera de Estomatología. Facultad de Ciencias Médicas «Raúl Dorticós Torrado», Cuba.

‡ Especialista de primer grado de Medicina Interna. Facultad de Ciencias Médicas «Raúl Dorticós Torrado».

§ Investigador agregado. Especialista de segundo grado de Estomatología General Integral. Facultad de Ciencias Médicas «Raúl Dorticós Torrado».

"I Investigador agregado. Doctorado en Filosofía con orientación en Psicología. Profesor investigador de la Universidad de Monterrey y Universidad del Valle de México. México.
}

Recibido: 14 Agosto 2020. Aceptado para publicación: 16 Agosto 2020. 
El brote inicial en un mercado local de mariscos en la ciudad de Wuhan, provincia de Hubei, en China a finales de diciembre del 2019, se ha extendido mundialmente, representando el mayor desafío sanitario en varias décadas, por lo que ha sido calificado por la Organización Mundial de la Salud (OMS) como una pandemia global desde el 11 de marzo del 2020.1,3,4,6,9-13

El 08 de enero del 2020, este nuevo coronavirus fue oficialmente anunciado como el patógeno causante de la COVID-19, por el centro chino para el control y prevención de la enfermedad (CDC, por sus siglas en inglés). ${ }^{5}$

En el principio, se cree en el origen animal, y más tarde se demuestra la transmisión posible de persona a persona. El 21 de enero del 2020, el COVID-19 fue clasificado en la categoría B de las enfermedades infecciosas de alta prioridad. El 30 de enero, la OMS declara el brote como una Emergencia de Salud Pública de Interés Internacional (PHEIC, por sus siglas en inglés). 5,9,10

En febrero del 2020, el COVID-19 había sido reconocido en 34 países, con un total aproximado de 81,000 casos confirmados y un total de 2,700 muertes. ${ }^{5,7}$

En marzo del 2020, la OMS reportaba que esta infección se diseminó sobre 100 países aproximadamente y causó más de 100,000 infecciones y más de 3,500 muertes. En esos momentos el ritmo de mortalidad era incierto, pero estimable entre $0.9-3 \% .{ }^{14}$

En mayo del 2020, aproximadamente 3.5 millones de casos y 250,000 muertes alrededor del mundo fueron

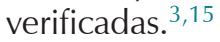

La potencial exposición al virus ha generado que la transmisibilidad del SARS-CoV-2 haya variado desde su aparición, hasta este momento en que continúa la propagación a los países de Europa, América y África, con una posibilidad de contagio por cada enfermo mayor de tres y menor de cinco personas sanas, dependiendo de las costumbres de interacción social y su medio cultural. ${ }^{10,15}$

La tasa total de casos globales fuera de China es de aproximadamente $4.5 \%$. Los cinco países con los más altos acumulados confirmados de casos en el mundo eran China, Italia, E.E. U.U., España y Alemania. ${ }^{4}$

Generar y conocer las medidas de prevención, identificación y gestión son pilares esenciales para cualquier profesional de salud en el intento de mitigar una mayor propagación de esta infección. ${ }^{16}$

El objetivo de la presente revisión es describir algunos parámetros para el diagnóstico de la COVID-19 y el papel del diagnóstico salival, de conocimiento relevante para el profesional en Estomatología.

La aproximación al diagnóstico del COVID-19 es todavía muy variable de región a región y de país a país.
Un camino clínico para implementar la estrategia diagnóstica más eficaz y pertinente es de gran importancia para establecer el control de la infección. ${ }^{9}$

Actualmente, es posible predecir el pronóstico utilizando síntomas, signos, y hallazgos imagenológicos, pero hay retos todavía en el camino. Los síntomas y signos, y los hallazgos imagenológicos son a menudo vistos más tarde en el curso de la enfermedad, por lo que la detección temprana puede ser difícil. ${ }^{9}$

La combinación de la historia clínica, las manifestaciones clínicas, la tomografía computarizada de tórax y la prueba viral tienen una alta sensibilidad (92-97\%). Globalmente, el diagnóstico temprano de esta infección es todavía un reto importante. $2,4,5,8,9,12,17$

El ensayo del ácido nucleico, el secuenciamiento del gen y el examen serológico (IgM y lgG), frotis de garganta o muestras de sangre han sido desarrolladas para establecer un diagnóstico patológico. ${ }^{9}$

\section{Prueba del ácido nucleico}

Posterior al brote inicial, muchas empresas de biotecnología exitosamente han desarrollado kits de detección del ácido nucleico, y el centro chino de Administración de Comida y de Droga (CFDA, por sus siglas en inglés) ha aprobado de manera urgente un lote de kits cuantitativos fluorescentes y sistemas de secuenciamento genéticos. La preocupación principal relacionada a la prueba del ácido nucleico estuvo relacionada a los falsos negativos. Para solucionar el problema de la baja eficacia de detección, algunas mejoradas pruebas rápidas de diagnóstico del ácido nucleico viral han sido inventadas. En particular, un papel de prueba del ácido nucleico, utilizado para la detección rápida del SARS-CoV-2 con una observación ocular desnuda en el lapso de tres minutos, ha sido exitosamente desarrollada. $2,4,8,12$

\section{Diagnóstico serológico}

Las pruebas serológicas están todavía en desarrollo, debido a la carencia de sensibilidad de un determinado número de pruebas, y de forma más importante, el retraso desde el tiempo en que se produce la infección hasta el desarrollo de anticuerpos. Estas pruebas, en cambio, pueden servir como herramienta útil para el análisis basado en la población, con propósitos epidemiológicos, mientras la prueba RT-PCR permanece como la mejor metodología para detectar las infecciones agudas., ${ }^{3,4}$

Las pruebas serológicas han sido usadas para diagnosticar infecciones con el SARS-CoV y el MERS-CoV, 
pero no son útiles en la fase aguda de la infección. Otra desventaja observada es la reactividad cruzada entre anticuerpos contra el SARS-CoV y los CoVs. ${ }^{14}$

Los pacientes con SARS-CoV-2 poseen respuestas agudas serológicas. Combinado con inmunocromatografía, oro coloidal y otras tecnologías, importantes reactivos de detección han sido desarrollados rápidamente. .,4,5,8,12

\section{Hallazgos de laboratorio}

En la fase temprana, la cuenta de glóbulos blancos es normal o disminuida, con una cuenta linfocitaria disminuida. Si la cuenta de linfocitos absoluta es 6, 14 .

Los hallazgos de laboratorio incluyen trombocitopenia, leucopenia, linfopenia y eosinopenia, según el caso y los últimos citados son los hallazgos de laboratorio primarios. Los dímeros $\mathrm{D}$, procalcitonina y los niveles CRP elevados pueden correlacionarse con mayor severidad de la enfermedad. Existen cambios en la albúmina, la deshidrogenasa láctica (DHL), los neutrófilos y la proteína $C$ reactiva (CRP) fueron vistos en las infecciones por SARS-CoV-2. $3,4,6,7,9,11,13-15$

El SARS-CoV-2 puede atacar principalmente a los linfocitos, en particular los linfocitos $\mathrm{T}$, al igual que lo hace el SARS-CoV. Las partículas del virus infectan las células, induciendo una tormenta de citoquinas en el cuerpo, generando una serie de respuestas inmunes y causando cambios en los glóbulos blancos periféricos y células inmunes. Consiguientemente, el bajo valor absoluto de los linfocitos puede ser utilizado como índice de referencia en el diagnóstico o para clasificar la severidad de las infecciones. ${ }^{9}$

Los biomarcadores potenciales para la severidad -niveles significativamente más altos del D-dímero, CRP, y procalcitonina- fueron asociados con una mayor severidad de la enfermedad. Un estudio informó que el tiempo de protrombina y los niveles de los dímeros $\mathrm{D}$ en la admisión eran más altos en la Unidad de Cuidados Intensivos ( $\mathrm{UCl}$ ), que en los pacientes fuera de ella. Las concentraciones en el plasma de IL-2, IL-7, IL-10, y TNF- $\alpha$ era más altas en los pacientes de la $\mathrm{UCl} .{ }^{9}$

Los pacientes que requieren admisión en la $\mathrm{UCl}$ tienen niveles más altos de factores estimulantes de colonias de granulocitos en el suero, factores estimulantes de colonias de macrófagos-granulocitos, proteína-10 interferón-inducible, proteína-1 quimiotáctica de monocitos, proteína-1A inflamatoria de macrófagos, factor de necrosis tumoral TNF- $\alpha$, más allá sugiriendo que la intensidad de la «tormenta de citocinas» modula la severidad de la enfermedad. ${ }^{15}$

\section{Diagnóstico molecular}

\section{Real-time reverse transcription PCR (RT-PCR) transcripción inversa en tiempo real}

El diagnóstico definitivo se realiza identificando la presencia del ARN viral del SARS-CoV-2, mediante la prueba de reacción en cadena de la polimerasa con transcriptasa inversa en tiempo real (RT-PCR, del inglés Real Time Reverse-Transcription-Polymerase-Chain-Reaction), la cual utiliza muestras provenientes tanto del tracto respiratorio superior (nasofaríngeo y orofaríngeo), como del tracto respiratorio inferior (esputo, aspirado endotraqueal o lavado broncoalveolar). Sin embargo, esta prueba ha mostrado problemas con la frecuencia de falsos negativos en las etapas tempranas de la enfermedad. Por tal razón, el diagnóstico y el aislamiento de muchos casos sospechosos hasta que no se tenga un análisis confirmatorio de la enfermedad, se apoya tanto en los signos clínicos como en la evidencia proveniente de los rayos $X$ y las tomografías axiales computarizadas (TAC) de los pulmones, para identificar las lesiones asociadas al COVID-19. . $^{-5,8,9,12,14,15,18-20}$

Las muestras de sospechosos de pacientes SARS-CoV-2 recogidas de la vía respiratoria superior (nasofaríngea y orofaríngea), vía respiratoria inferior (esputo expectorado, aspirado endotraqueal o lavado bronqueoalveolar), la sangre y las heces pueden ser diagnosticadas por RT-PCR. Las dos regiones de secuencia (ORF1b y N) diseñadas, basadas en la primera secuencia de acceso público en GenBank, han sido seleccionadas para primer y diseños de sonda, los cuales son altamente conservados entre Sarbecovirus. El ensayo del gen $\mathrm{N}$ es 10 veces más sensible que el ensayo del gen ORF1b, en detectar especímenes clínicos positivos. ${ }^{6}$

Los métodos existentes de PCR tienen especificidad muy buena, pero sensibilidad baja, significando este dato que los resultados negativos no pueden excluir la presencia de SARS-CoV-2. Además, la contaminación de las muestras del laboratorio, causada por la carencia de control, puede dirigir la confirmación de falsos resultados positivos. Además, las pruebas RT-PCR pueden ser falsamente negativas debido a insuficientes materiales virales o errores operacionales. Algunos pacientes con resultados negativos en el RT-PCR pueden presentar hallazgos positivos al realizarse la CT para detectar la COVID-19, esto significa que los resultados al PCR pueden ayudar a la evaluación y el diagnóstico clínico, pero la posibilidad de la enfermedad no puede ser confirmada o descartada. Para el individuo con una sospecha clínica alta, pero ne- 
gativa al RT-PCR, una combinación del escaneo con CT y pruebas de frotis repetidos pueden ser útiles. ${ }^{6}$

\section{Técnica SHERLOCK}

La técnica CRISPR-based SHERLOCK (Specific Highsensitivity Enzymatic Reporter UnLOCKing) permite una detección portátil, múltiple y ultrasensible de ARN o ADN de muestras clínicamente pertinentes. Estos ensayos están conformados con preamplificación recombinada mediada por polimerasa del ADN o ARN y proporcionan resultados en menos de una hora, sin requerir instrumentación elaborada, pero aún necesitan ser confirmados con muestras de pacientes. ${ }^{6}$

Basado en la secuencia de ARN del nuevo coronavirus, los investigadores cuidadosamente diseñaron dos guías ARNs, uno reconociendo el gen $\mathrm{S}$ del nuevo coronavirus y el otro reconociendo el gen Orf1ab. Para maximizar la exactitud de la detección, los científicos han seleccionado secuencias que son más específicas para el nuevo coronavirus. De este modo, la interferencia de otros genomas de virus respiratorios también puede ser minimizada. Teóricamente, mientras el ARN corresponde al nuevo coronavirus en la muestra, el ARN de guía con exactitud puede reconocer y activar la proteína Cas13a para unirse a ella. Es una enzima muy interesante; una vez activada la Cas13a, corta de manera indiscriminada e intensamente cualquier otra molécula de ARN que encuentre. ${ }^{6}$

\section{Sistema CRISPR/Cas13}

El Cas13-based SHERLOCK (Specific High-sensitivity Enzymatic Reporter unLOCKing) es una plataforma que ha sido ampliamente utilizada para detectar el virus del Zika (ZIKV) y el virus del dengue (DENV) en muestras de pacientes en concentraciones tan bajas como una copia por microlitro. Recientemente, esta tecnología ha sido usada para detectar el virus del SARS-CoV-2. Aun así, este sistema tiene que ser verificado porque no ha sido probado en muestras clínicas de pacientes de $\mathrm{CO}$ VID-19. ${ }^{2,4,5,8,12}$

\section{Diagnósticos salivales del COVID-19}

De manera general, las muestras apropiadas incluyen las vías aéreas superiores e inferiores, la sangre, las heces, la orina y las secreciones conjuntivales. 3,21

La utilización de la saliva como una muestra viable para la detección de la COVID-19 es una fuente robusta, debido a la extracción del ARN viral. ${ }^{19}$
La saliva es un espécimen prometedor para el diagnóstico y monitoreo de la infección del virus SARS-CoV-2. Los virus han sido detectados en estudios mediante RTqPCR y en la saliva autorrecolectada, mostrando altas cargas virales en la primera semana después del inicio de los síntomas, con una tendencia gradual a decrecer en los días subsecuentes. Además, mediante el cultivo viral se confirmó que la mayor carga viral se presenta en la primera semana, pero que el ARN viral aún podría detectarse en muestras de saliva y orofaríngeas en un tercio de los pacientes, durante 20 días o más desde el inicio de los síntomas. ${ }^{2,16,17,19,22}$

Aunque la COVID-19 es diagnosticada como una enfermedad respiratoria, se puede constatar la excreción viral en las heces y en la saliva. El ARN viral puede persistir en las heces, incluso después de la mejora de los síntomas respiratorios, de dos a cuatro semanas. ${ }^{10,21,22}$

La expresión de ACE-II en humanos se ha identificado en la lámina basal del epitelio de la mucosa oral, así como también en el epitelio de la lengua y piso de boca, y también en el epitelio del conducto de las glándulas salivales en macacos Rhesus (Macaca mulatta) donde se demostró la infección con el SARS-CoV. Estos datos parecen corresponder con la presencia de niveles mayores del virus en la cavidad oral y la orofaringe, que en la nasofaringe, lo cual permite sugerir que la liberación del virus es eficiente en la saliva, aunque la saliva total contiene secreciones que provienen del tracto respiratorio, del líquido crevicular y de las glándulas salivales, por lo cual se podría presumir que el origen de las partículas virales detectadas con las pruebas de laboratorio podrían tener un origen oral cuestionable. Sin embargo, se ha detectado este virus en muestras de saliva en pacientes sin fiebre y con carga viral alta desde los primeros días después de la infección. 2,3,15,18

Por lo tanto, la saliva representa una muestra de fácil obtención, que no requiere el contacto cercano con el paciente, quien ejecuta instrucciones sencillas a distancia para escupir la saliva total en un recolector estéril, preparado para tal fin con solución inhibidora de ARNasa, que posteriormente se procesará mediante RT-PCR para la detección del virus. Es decir, se trata de un método que se puede emplear para hacer la detección temprana del virus y con menor riesgo de transmisión nosocomial para el personal de la salud, lo que facilitaría la implementación oportuna de las medidas de prevención y control de los casos positivos. ${ }^{17,18}$

Como ventajas de las pruebas salivales, se puede afirmar que éstas no son invasivas, son más económicas, rápidas y seguras de administrar, en comparación con las 
pruebas serológicas. Los valores diagnósticos son ofrecidos en tiempo real. No hay necesidad de entrenar al personal médico, las muestras pueden ser obtenidas fácilmente, y la colección y escaneo pueden hacerse en el hogar. Se minimiza el riesgo de infección cruzada. Las muestras son más económicas de manipular y almacenar, requiriendo menos manipulación durante los procedimientos diagnósticos, en comparación con las pruebas serológicas. Además, existe disponibilidad comercial de los ensayos y la saliva puede ser manipulada más fácilmente que la sangre, ya que no forma coágulos. ${ }^{17,19}$

Teóricamente, el diagnóstico puede establecerse por las plataformas de diagnóstico salivales. Algunos virus han sido identificados 29 días después de la infección, sugiriendo que una plataforma no invasiva para diferenciar los biomarcadores usando la saliva, puede incrementar la detección de la enfermedad. La colección nasofaríngea u orofaríngea no es confortable para el paciente, y puede causar sangramiento, particularmente en pacientes con trombocitopenia. Se plantea que sólo 28\% de los pacientes con COVID-19 producen esputo proveniente del tracto respiratorio inferior, sugiriendo una limitante significativa como espécimen de la evolución diagnóstica. ${ }^{17,19,20}$

La saliva tiene un alto margen de consistencia, de más de $90 \%$, con especímenes nasofaríngeos en la detección de los virus respiratorios, incluyendo los coronavirus. ${ }^{19}$

Existen tres vías para la existencia del COVID-19 en la saliva: ${ }^{17,19,20}$

1. COVID-19 en el tracto respiratorio superior e inferior, entrando en la cavidad oral junto con las gotas liquidas que son regularmente intercambiadas con estos órganos.

2. COVID-19 en la sangre entrando en la boca mediante el líquido crevicular en la encía.

3. Infección de las glándulas salivales mayores y menores con la liberación subsecuente de las partículas en la saliva mediante los conductos salivares.

Las células del epitelio glandular pueden estar afectadas por este virus, después de la infección, hecho comprobado en los monos Rhesus. Este dato indica que las células de las glándulas salivales pueden ser una fuente clave para este virus en la saliva. ${ }^{17,20}$

El diagnóstico de las infecciones virales en la saliva depende de los biomarcadores, tales como el ADN y el ARN virales, antígenos y anticuerpos. El instituto científico Raffaele en Milán, usa un test llamado prueba salival de anticuerpos de la hepatitis C OraQuick ${ }^{\circledR}$. El dengue tiene antígenos detectables en la saliva, y la IgM y la
IgG proveen una forma efectiva para diagnosticar esta enfermedad, con sensibilidad de $92 \%$ y especificidad de $100 \%$. Hay estudios que afirman que la saliva parece más sensible que la sangre para la detección de otros virus como son el HHV-6 o el CMVH. Las pruebas salivales usando una reacción en cadena de la polimerasa, para la detección del virus del papiloma humano (HPV, human papillomavirus), han sido establecidas. ${ }^{19}$

El desarrollo de la inmunoglobulina A secretoria específica del SARS-CoV (SIgA) en la saliva de los modelos animales, ha sido demostrada previamente. Tomando en cuenta la similaridad entre ambas cadenas, el diagnóstico salival del COVID-19 puede ser logrado usando anticuerpos específicos contra este virus. Como resultado, la saliva juega un rol crucial en la transmisión de humano a humano, y los diagnósticos salivales pueden crear una plataforma efectiva y simple para el diagnóstico definitivo. ${ }^{17}$

Uno de los mecanismos más importantes para el desarrollo del síndrome de dificultad respiratoria aguda en esta enfermedad es la «tormenta de citosinas», la cual libera una larga cantidad de citocinas proinflamatorias (IFN- $\alpha$, IFN- $\gamma$, IL-1b, IL-6, IL-12, IL-18, IL-33, TNF- $\alpha$, TGF- $\beta$, etcétera) y quimiocinas (CCL2, CCL3, CCL5, CXCL8, CXCL9, CXCL10, etcétera). ${ }^{19}$

Este incremento en las citocinas proinflamatorias en pulmón y otros órganos de pacientes con SARS, y el síndrome de respuesta inflamatoria sistémica proporciona un mecanismo potencial para la falla multiorgánica. ${ }^{23}$

\section{Hallazgos imagenológicos}

Los hallazgos imagenológicos de la COVID-19 pueden ser variables y cambiar rápidamente. Los exámenes radiológicos son importantes para detección temprana, control y evaluación de la COVID-19. Normalmente no hay ningún hallazgo anormal en la fase temprana con radiografía digital (DR). Los pacientes con enfermedad confirmada por laboratorio sin manifestaciones radiológicas también han sido reportados. ${ }^{9}$

La radiografía de tórax y las tomografías computarizadas (TC) de tórax son herramientas importantes para el diagnóstico de la COVID-19 en la práctica clínica. La inteligencia artificial (IA) ha sido usada con exactitud para interpretar las imágenes de las TC de los casos sospechosos de coronavirus y la tasa de exactitud de los resultados de su análisis logra 96\%, mejorando por mucho la eficacia diagnóstica de los hallazgos imagenológicos. Esta técnica ya está siendo utilizada en la práctica clínica. 2,4,5,8,12,17

La tomografía computarizada de tórax anormal ha sido usada para diagnosticar la mayoría de los casos de 
COVID-19, indicando que la neumonía es la manifestación más común de la enfermedad. Por tanto, estas imágenes son ampliamente utilizadas en el diagnóstico temprano, aunque la TC no puede distinguir la COVID-19 de otras neumonías virales. Los hallazgos típicos incluyen opacidades multifocales en vidrio esmerilado distribuidas periféricamente (GGOs, por sus siglas en inglés) con consolidaciones irregulares. Números crecientes, alcance y densidad de GGOs en las TC, se correlacionan con la progresión de la enfermedad. 2,4-6,8,9,12,15,17

\section{BIBLIOGRAFÍA}

1. Martí SD, Fernández PC, Felix MA, Delgado CF, Estébanez MM, Álvarez AS. Aspectos cardiológicos relevantes en la infección COVID-19. RIECS 2020, 5. Disponible en: https://doi. org/10.37536/RIECS.2020.5.1.203.

2. Peng X, Xu X, Li Y, Cheng L, Zhou X, Ren B. Transmission routes of 2019-nCoV and controls in dental practice. Int J Oral Sci. 2020; 12 (1): 9. Available in: https://doi.org/10.1038/s41368-020-0075-9.

3. Dingemans AC, Soo RA, Jazieh AR, Rice SJ, Kim YT, Teo L et al. Treatment guidance for patients with lung cancer during the coronavirus 2019 pandemic. J Thorac Oncol. 2020; 15 (7): 11191136. Available in: https://doi.org/10.1016/j.jtho.2020.05.001.

4. Jin Y, Yang H, Ji W, Wu W, Chen S, Zhang W, Duan G. Virology, Epidemiology, pathogenesis, and control of COVID-19. Viruses. 2020; 12 (4): 372. Available in: https://doi.org/10.3390/v12040372.

5. Meng L, Hua F, Bian Z. Coronavirus disease 2019 (COVID-19): emerging and future challenges for dental and oral medicine. J Dent Res. 2020; 99 (5): 481-487. Available in: https://doi. org/10.1177/0022034520914246.

6. Kang S, Peng W, Zhu Y, Lu S, Zhou M, Lin W et al. Recent progress in understanding 2019 novel coronavirus (SARS-CoV-2) associated with human respiratory disease: detection, mechanisms and treatment. Int J Antimicrob Agents. 2020; 55 (5): 105950. Available in: https://doi.org/10.1016/j.ijantimicag.2020.105950.

7. Xiao AT, Tong YX, Gao C, Zhu L, Zhang YJ, Zhang S. Dynamic profile of RT-PCR findings from 301 COVID-19 patients in Wuhan, China: A descriptive study. J Clin Virol. 2020; 127: 104346. Available in: https://doi.org/10.1016/j.jcv.2020.104346.

8. Wu C, Zhang Z, Mo Y, Wang D, Ning B, Xu P et al. Recommendations for control and prevention of infections for pediatric orthopedics during the epidemic period of COVID-19. World Journal of Pediatric Surgery. 2020, 3 (1): e000124. Available in: https://doi. org/10.1136/wjps-2020-000124.

9. Xu G, Yang Y, Du Y, Peng F, Hu P, Wang R et al. Clinical pathway for early diagnosis of COVID-19: updates from experience to evidencebased practice. Clin Rev Allergy Immunol. 2020; 59 (1): 89-100. Advance online publication. Available in: https://doi.org/10.1007/ s12016-020-08792-8.

10. She J, Liu L, Liu W. COVID-19 epidemic: disease characteristics in children. J Med Virol. 2020; 92 (7): 747-754. Available in: https:// doi.org/10.1002/jmv.25807.

11. Wang D, Yin Y, Hu C, Liu X, Zhang X, Zhou et al. Clinical course and outcome of 107 patients infected with the novel coronavirus, SARS-CoV-2, discharged from two hospitals in Wuhan, China. Crit Care. 2020; 24 (1): 188. Available in: https://doi.org/10.1186/ s13054-020-02895-6.
12. He F, Deng Y, Li W. Coronavirus disease 2019: What we know? J Med Virol. 2020; 92 (7): 719-725. Available in: https://doi. org/10.1002/jmv.25766.

13. Rameshrad M, Ghafoori M, Mohammadpour AH, Nayeri $M$, Hosseinzadeh $\mathrm{H}$. A comprehensive review on drug repositioning against coronavirus disease 2019 (COVID19). NaunynSchmiedeberg's Arch Pharmacol. 2020; 393: 1137-1152. Advance online publication. Available in: https://doi.org/10.1007/s00210020-01901-6.

14. Zimmermann $P$, Curtis N. Coronavirus infections in children including COVID-19: an overview of the epidemiology, clinical features, diagnosis, treatment and prevention options in children. Pediatr Infect Dis J. 2020; 39 (5): 355-368. Available in: https:// doi.org/10.1097/INF.0000000000002660.

15. Boukhris M, Hillani A, Moroni F, Annabi MS, Addad F, Ribeiro MH. Cardiovascular implications of the COVID-19 pandemic: a global perspective. The Can J Cardiol. 2020; 36 (7): 1068-1080. Available in: https://doi.org/10.1016/j.cjca.2020.05.018.

16. Sigua-Rodríguez EA, Bernal-Pérez JL, Lanata-Flores AG, SánchezRomero C, Rodíguez-Chessa J, Haidar ZS et al. COVID-19 y la Odontología: una revisión de las recomendaciones y perspectivas para Latinoamérica. Int J Odontostomat. 2020; 14 (3): 299-309.

17. Baghizadeh FM. What dentists need to know about COVID-19. Oral Oncol. 2020; 105: 104741. Available in: https://doi.org/10.1016/j. oraloncology.2020.104741.

18. Pérez-Domínguez M, Pérez-Ybarra L. SARS-CoV-2 en saliva: potencial vía de contagio e implicaciones en el tratamiento del paciente odontológico. Odous Científica. 2020; 21(1): 77-88.

19. Sri Santosh T, Parmar R, Anand H, Srikanth K, Saritha M. A review of salivary diagnostics and its potential implication in detection of Covid-19. Cureus. 2020; 12 (4): e7708. Available in: https://doi. org/10.7759/cureus.7708.

20. Alharbi A, Alharbi S, Alqaidi S. Guidelines for dental care provision during the COVID-19 pandemic. Saudi Dent J. 2020; 32 (4): 181-186. Advance online publication. Available in: https://doi. org/10.1016/j.sdentj.2020.04.001.

21. Mao R, Qiu Y, He JS, Tan JY, Li XH, Liang J, et al. Manifestations and prognosis of gastrointestinal and liver involvement in patients with COVID-19: a systematic review and meta-analysis. Lancet Gastroenterol Hepatol. 2020; 5 (7): 667-678. Available in: https:// doi.org/10.1016/S2468-1253(20)30126-6.

22. Manus JM. Symptômes gastro-intestinaux initiaux, autres signes de Covid-19. Rev Francoph Lab. 2020; (522): 16-17. Available in: https://doi.org/10.1016/S1773-035X(20)30153-2.

23. Barbagelata A, Perna ER, Piskorz D, Lorenzatti A. Prevención del colapso del sistema de salud en pacientes cardiovasculares con Covid-19: el rol del cardiólogo en la reducción de la sobrecarga de las unidades de cuidados intensivos con el advenimiento del frío en América del Sur. Rev Fed Arg Cardiol. 2020; 49 (Reporte COVID19): 4-12.

\section{Correspondencia:}

\section{Francisco Cázares de León}

Paseo de la Cordillera Núm. 3113,

Col. Cumbres 2 Sector, 64610,

Monterrey, Nuevo León, México,

Tel. 83000831 ,

E-mail: francisco.cazares@udem.edu, fclsalud@gmail.com 